\title{
How to Grow a Lung: Applying Principles of Developmental Biology to Generate Lung Lineages from Human Pluripotent Stem Cells
}

\author{
Briana R. Dye ${ }^{1}$ Alyssa J. Miller ${ }^{2,3} \cdot$ Jason R. Spence ${ }^{1,2,3,4}$
}

Published online: 18 April 2016

(c) The Author(s) 2016. This article is published with open access at Springerlink.com

\begin{abstract}
The number and severity of diseases affecting human lung development and adult respiratory function has stimulated great interest in new in vitro models to study the human lung. This review summarizes the most recent breakthroughs deriving lung lineages in a dish by directing the differentiation of human pluripotent stem cells. A variety of culturing platforms have been developed, including two-dimensional and three-dimensional (organoid) culture platforms, to derive specific cell types and structures of the lung. These stem cell-derived lung models will further our understanding of human lung development, disease, and regeneration.
\end{abstract}

Keywords Directed differentiation - Human pluripotent stem cell · Organoid · Lung development · Airway · Alveoli

This article is part of the Topical collection on Application of Stem Cells in Endoderm Derivatives.

Jason R. Spence

spencejr@umich.edu

Briana R. Dye

rockichb@umich.edu

Alyssa J. Miller

ajanemil@umich.edu

1 Department of Cell and Developmental Biology, University of Michigan Medical School, Ann Arbor, Michigan 48109, USA

2 Department of Internal Medicine, University of Michigan Medical School, Ann Arbor, Michigan 48109, USA

3 Department of Cell and Molecular Biology, University of Michigan Medical School, Ann Arbor, Michigan 48109, USA

4 Center for Organogenesis, University of Michigan Medical School, Ann Arbor, Michigan 48109, USA

\section{Introduction}

The lungs are the main organ of the respiratory system and function to exchange gas, taking in oxygen and expelling carbon dioxide. The lungs are organized in a branched, tree-like structure that has two major anatomical features, the conducting airways and alveoli. The conducting airways are arranged in a series of tubes that become progressively smaller as they move gas into the alveoli. The conducting airways start as a singular tube, the trachea, which splits into two main bronchi. The main bronchi then further split and branch into smaller airway tubes called the conducting bronchioles. The walls of the conducting airways consist of epithelial cell types surrounded by supporting mesenchyme including fibroblasts, smooth muscle, cartilage, vasculature, and neurons extending the length of the airways (Fig. 1). Just before the bronchioles terminate they form a small tube called the alveolar duct, which leads into the thin sac of cells that make up the alveoli. The alveolar epithelium is tightly associated with a capillary network in order for efficient gas exchange to take place.

In order to accommodate diverse functions, the human lung possesses several specialized cell types. The conducting airways are predominated by three epithelial cell types: ciliated, goblet, and basal cells, with ciliated cells being the most dominant cell type in human airways [1]. Ciliated cells have multiple cilia that beat in a synchronous rhythm in order to maintain the flow of mucus across the airway epithelium [2,3]. Goblet cells are secretory cells that secrete mucus on the surface of the airway [4]. The basal cells are the adult stem cells of the airways [5-9]. In humans, basal cells line the main bronchi and large bronchioles, but begin to decrease in number in the smaller bronchioles toward the alveoli $[1,8,10,11]$. Other, sparse populations of cells in the airway include club cells, which 
are secretory cells that secrete lubricating glycosaminoglycans and antimicrobial peptides into airways [12], and pulmonary neuroendocrine cells which are innervated on the basal surface and store proteins that are released under a physiological stimulus such as hypoxia [13-16]. The alveolar epithelium is composed of two epithelial cell types, alveolar epithelial type I cells (AECI) and alveolar epithelial type II cells (AECII) (Fig. 1). AECIs form a thin, squamous epithelium that covers the majority of the alveolar surface and exchanges gas with neighboring capillaries through diffusion [17-20]. AECIIs have a cuboidal shape and secrete surfactant proteins to reduce the surface tension of the alveolar sacs, allowing them to expand and contract without collapsing as breathing takes place [18, 20-22].

The complex architecture of the adult lung is established during early embryonic development. The epithelium of the lung is derived from the embryonic endoderm while much of the structural support of the lung, including fibroblasts, smooth muscle, and cartilage, is derived from the embryonic mesoderm. Lung development begins as two buds forming off of a tube of endoderm surrounded by mesoderm called the gut tube. The gut tube gives rise to the entire gastrointestinal tract and associated organs, including lungs, thyroid, liver, and pancreas [23-26]. The primary lung buds emerge from the gut tube, invade the surrounding mesoderm and continue to elongate in tight association with the mesoderm throughout development. The lung buds then undergo branching morphogenesis, in which epithelial bud tips continuously bifurcate, forming new branches, until a stereotyped tree-like pattern is formed [27]. During branching, a proximal-distal lung axis is established, with proximal lung epithelium giving rise to conducting airways, and the distal tips eventually terminally differentiating into the alveolar sacs (for more detailed reviews see: $[28,29])$.

Since lung development is so critical for neonatal life, it has been the focus of intense study. In addition, lung diseases are prevalent and can be caused by environmental exposure to pathogens or damaging toxins; however, in many cases, chronic lung diseases are often a result of both genetics and environment. Much of our understanding of aberrant lung development and adult disease has resulted from using animal models and cell culture systems. These approaches have yielded a tremendous amount of insight into the mechanisms that cause disease; however, there are many aspects of human biology and disease that are not reflected in animal models or cell culture systems. In order to address the need for physiologically relevant human model systems, the past decade has seen several fields turn to human pluripotent stems cells (hPSCs), which include both embryonic stem cells and induced pluripotent stem cells, in an attempt to generate specific cell types and even

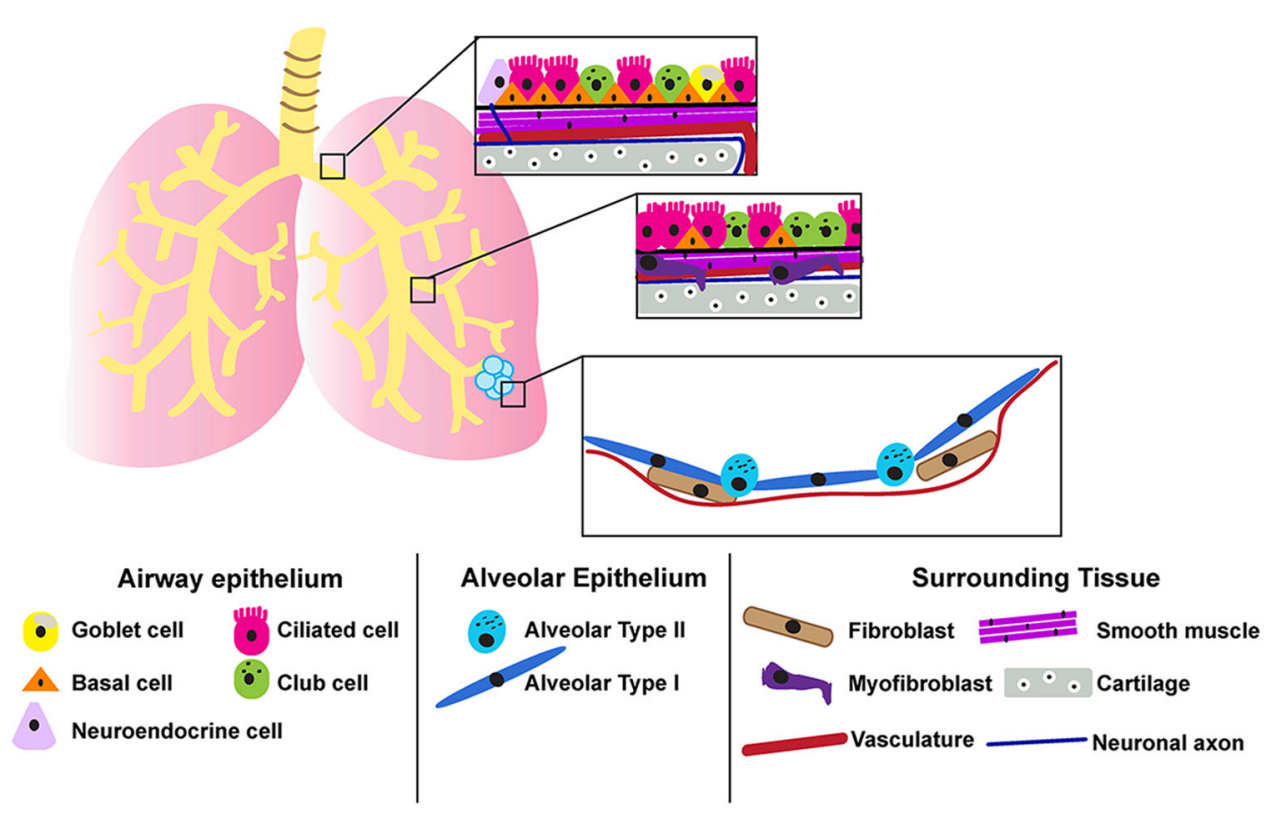

Fig. 1 A diagram summarizing the lung cell types in and surrounding the airways and alveoli. Upper airways are surrounded by cartilage (brown) and smooth muscle (pink). The upper airway epithelium is lined with basal cells (orange) with ciliated (pink), goblet (yellow), club (green), and neuroendocrine (light purple) cells adjacent to the basal cells facing toward the lumen of the airway. The lower airways possess less basal cells and consist of mostly ciliated and club cells with surrounding tissues consisting of smooth muscle, myofibroblasts (purple), and patches of cartilage. Vessels (red line) and neurons (blue line) line both the upper and lower airways. The alveolar sacs consist of elongated AECI (light blue) and cuboidal AECII cells (dark blue) that are lined with thin vessels in order for gas exchange to occur with few fibroblasts scattered outside the alveolar sac. Note that only the most abundant lung cell types are depicted (Color figure online) 
complex organ-like tissue in vitro [30••, 31-34]. Indeed, the lung field has seen rapid growth in the number of exciting reports generating lung tissue in vitro $\left[35^{\bullet}, 36^{\bullet}\right.$, $37 \bullet, 38 \bullet, 39 \bullet, 40 \bullet, 41 \bullet, 42 \bullet, 43 \bullet, 44 \bullet, 45 \bullet$. In this review article, we will introduce the developmental biology framework that forms the basis used by many groups attempting to differentiate lung tissue from hPSCs. We will then highlight successful efforts to generate 'lung tissue in a dish,' and discuss the implementation of these varied human model systems, followed by a discussion of their strengths and limitations.

\section{Endoderm and Anterior Foregut Specification}

hPSC-derived tissues are often generated using directed differentiation, a process that aims to recapitulate the signals that drive cell or organ-specific differentiation in the

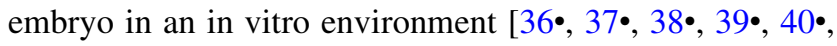
$41^{\bullet}, 42^{\bullet}, 43^{\bullet}, 44^{\bullet}, 45^{\bullet}, 46^{\bullet}$ ]. In the case of the lung, this entails a series of sequential steps to derive endoderm, then anterior foregut endoderm and induction of lung progenitor cells, followed by lung-specific cell type differentiation and maturation. It has long been appreciated that Nodal signaling is necessary to form definitive endoderm during gastrulation in animal models, and is also sufficient to convert non-endodermal lineages (ectoderm) into endoderm in early embryonic tissue [26, 46-50]. In cell culture, ActivinA, which is a $\operatorname{Tgf} \beta$ superfamily member, stimulates the same signaling pathways as Nodal and has been widely used to successfully induce definitive endoderm differentiation from hPSCs [30••, 34, 39•, 51].

Following gastrulation, the embryo undergoes a series of morphological movements that give rise to the gut tube, which is an endodermal tube surrounded by mesoderm. Embryo and gut tube patterning are guided by a multitude of secreted proteins that both stimulate and inhibit different signaling pathways in order to establish the domains of the gut tube, referred to as the foregut, midgut, and hindgut which form along the anterior-posterior axis of the embryo (for more detailed reviews, see: [26, 52]).

Several secreted inhibitors help to establish the anterior identity of the foregut endoderm, which will give rise to the lungs and thyroid. As the endoderm migrates to form the gut tube during gastrulation, cells at the anterior pole of the embryo encounter secreted Nodal inhibitors Lefty1 and Cerberus-like (Cerl) and BMP inhibitors Chordin and Noggin, which inhibit posterior patterning [53-55]. In Xenopus laevis, the Wnt antagonist Sfrp5 is secreted from the endoderm to maintain foregut identity [56]. In line with embryonic regulation of anterior endoderm patterning, an activator/inhibitor screen conducted in hPSC-derived endoderm that included inhibitors and activators of Nodal,
Hedgehog (HH), WNT, RA, BMP, and TGF $\beta$ signaling pathways identified that inhibition of the BMP and TGF $\beta$ signaling pathways robustly induced anterior foregut genes [57••]. The observation that inhibition of BMP and TGF $\beta$ ("dual smad inhibition") potently stimulates a foregut endoderm fate has been widely adopted by the lung differentiation field $\left[38^{\bullet}, 39^{\bullet}, 40^{\bullet}, 41^{\bullet}, 45 \bullet, 57 \bullet \bullet\right]$ Interestingly, although secreted Wnt inhibitors help maintain foregut identity in vivo, inhibition of Wnt signaling is not necessary to induce foregut endoderm in vitro $[36 \bullet, 37 \bullet$, $39 \bullet, 40 \bullet, 41 \bullet, 45 \bullet, 57 \bullet \bullet]$.

\section{Morphogenesis in a Dish}

In hPSC-derived endoderm cultures, it has been shown that stimulating WNT and FGF signaling pathways (via WNT3A and FGF4) cause self-aggregation of three-dimensional cell clusters, called spheroids, that delaminate from the tissue culture monolayer [34, 39•, 51, 58]. While the mechanisms driving three-dimensional spheroid formation in endoderm cultures is not known, it is interesting to speculate that FGF4 and WNT are acting, as they do in vivo, to drive cellular reorganization and migration [5963].

In addition to driving three-dimensional morphogenesis in a dish, WNT3A and FGF4 are potent stimulators of the CDX2+ intestinal lineage in vitro [34]. However, simultaneous stimulation of WNT/FGF signaling in combination with the in vitro anterior foregut promoting conditions (dual smad inhibition), caused spheroids to take on an anterior foregut fate, including the expression of, NKX2.1, PAX8, and SOX2 [39॰]. Taken together, these studies demonstrated that hPSC-derived definitive endoderm can be directed to become anterior foregut endoderm by mimicking in vivo patterning signals.

\section{Lung Induction}

During early development, the lung primordium forms along the ventral anterior foregut and expresses the transcription factor Nkx2.1, which is required for lung fate [64, 65]. Extensive study of lung specification has identified several signaling pathways that are critical for this process, including Fgf, Bmp, Wnt, Hedgehog (Hh), and RA signaling pathways (reviewed in $[28,66]$ ). Bmp signaling to the anterior-ventral foregut endoderm is important to prime the lung domain by inhibiting Sox2, allowing lung specification to take place $[66,67]$. Additionally, Fgf2 secreted from the cardiac mesoderm, which sits adjacent to the ventral foregut, and Fgf10 secreted from the surrounding lung mesoderm are necessary for $\mathrm{Nkx} 2.1$ expression and 
lung formation [68-71]. Similarly, Wnt ligands signaling from the lung mesoderm to the ventral foregut endoderm are also necessary for Nkx2.1 expression and lung induction [72-75]. RA promotes $\mathrm{Nkx} 2.1$ expression and lung bud formation in part by inhibiting TGF $\beta$ and the Wnt antagonist Dkk1, allowing Fgf and Wnt signaling to occur $[76,77]$. Hh signaling is also critical for lung development, as concurrent deletion of the $\mathrm{Hh}$ signaling transcription effectors Gli2 and Gli3 leads to lung agenesis [78].

Since the events required for lung induction are complex and involve multiple signaling pathways that are controlled in very tight temporal and spatial manner in vivo, translating these developmental paradigms in a dish has proven to be challenging. However, many groups have generated hPSC-derived NKX2.1+ lung progenitor populations with varying levels of efficiency (Fig. 2a-c) [35•, 36•, 37•, 38•, $\left.39 \bullet, 40^{\bullet}, 41^{\bullet}, 43^{\bullet}, 44^{\bullet}, 45^{\bullet}\right]$. The majority of methods to derive lung tissue in vitro have treated endoderm, or anterior foregut endoderm, in monolayer cultures with FGFs, BMPs, WNTs, and RA to induce NKX2.1+ endoderm. Approaches to induce three-dimensional lung organoids have used a combination of factors to promote spheroid formation by activating FGF4 and WNT signaling, while simultaneously specifying foregut with dual smad inhibition and inducing lung.

To date, the field has primarily focused on differentiating lung epithelial cells from hPSCs, however, the lung mesenchyme also plays a critical role in sending and receiving signals and physically interacting with the lung epithelium during early development [28]. Given the important contribution of the mesenchyme to lung development and lung function, modeling this aspect of lung development in vitro represents a significant opportunity for furthering the field.

\section{Proximal-Distal Patterning in the Lung}

Following the induction of an Nkx2.1+ field of lung progenitor cells, the primordial lung buds undergo branching morphogenesis, giving rise to a patterned epithelium that consists of Sox $2+$ proximal airways and Sox $9+/ \operatorname{Id} 2+/$ Nmyc+ actively branching epithelium that will eventually differentiate, giving rise to the alveoli [79-82]. Diffusible growth factors including Bmp4, Fgfs, and Wnts are essential for establishing and maintaining the proximaldistal pattern of the lung. Bmp 4 and Wnt $2 / 2 \mathrm{~b}$ expression in the distal mesenchyme and Wnt7a originating in the distal epithelium signal to the distal epithelium and are essential for maintaining the distal epithelium and promoting branching [83-87]. In mice, blocking Wnt signaling by conditional loss of $\beta$-Catenin from the epithelium results in disrupted branching and expansion of the proximal epithelium [84, 88]. Similarly, Fgf7 and Fgf10 signaling from the distal mesenchyme to the epithelium promotes epithelial growth and branching, and Fgf10 plays a role in maintaining distal progenitor cells during branching morphogenesis [89-93]. RA promotes branching by increasing Fgf10 expression in the lung mesenchyme [94]. Once the branching program is complete, the distal epithelium differentiates into the alveolar cell types, AECI and AECII. In the mouse lung, both of these cell types arise from a distal bipotent progenitor [95], however; the factors that regulate cell fate choice and differentiation of alveolar cell types in the embryo are not well understood.

Efforts to derive distal and alveolar cell types in vitro have attempted to recapitulate the signaling factors active around distal buds during branching in the developing embryo. To date, Huang et al. have discerned the most efficient methods to generate AECI and AECII cells from hPSCs (Fig. 2a) [38•]. hPSC-derived foregut endoderm was cultured with Chir99021 (a GSK3 $\beta$ antagonist that stabilizes $\beta$-catenin), BMP4, FGF10, FGF7, and RA for 15 days at which time the cells were cultured with Chir99021, FGF10, and FGF7 for an additional 25 days, followed by treatment with dexamethasone, cAMP, and isobutylmethylxanthine (DCI), which stimulates alveolar cell-specific gene expression in vitro [35•, 96]. Interestingly, dexamethasone is administrated to premature infants to accelerate fetal lung maturation, which results in enhanced surfactant secretion from AECIIs [97, 98]. These sequential steps resulted in cells expressing AECII protein SFTPB in over $50 \%$ of all cells in the culture, with some SFTPB + cells displaying lamellar bodies and functional release and uptake of surfactant protein. These factors also induced cells to express AECI cell-specific markers. While these cells displayed the flat, elongated nuclei typical of AECI cells, they did not exhibit elongated cell bodies or form multi-cellular sac-like alveolar structures [38•]. Others have found that seeding NKX2.1+ lung progenitors onto human lung extracellular matrix (ECM) proteins significantly enhanced AECI and AECII cell differentiation, indicating that both the physical and chemical environments are important for alveolar differentiation in vitro [42•].

More recently, attempts have been made to differentiate three-dimensional lung-like tissues in vitro by recreating the embryonic distal lung environment in a dish (Fig. 2b) [39•]. Three-dimensional foregut spheroid cultures grown in an extracellular matrix (Matrigel) with high concentrations of FGF10 led to the formation of lung organoids. Lung organoids possessed a distal alveolar cell population that expressed bipotent progenitor markers including SFTPC, HOPX, and SOX9 [95] with a few cells expressing mature AECI and AECII markers, PDPN and SFTPB, respectively. Alveolar cell types were found in distinct 


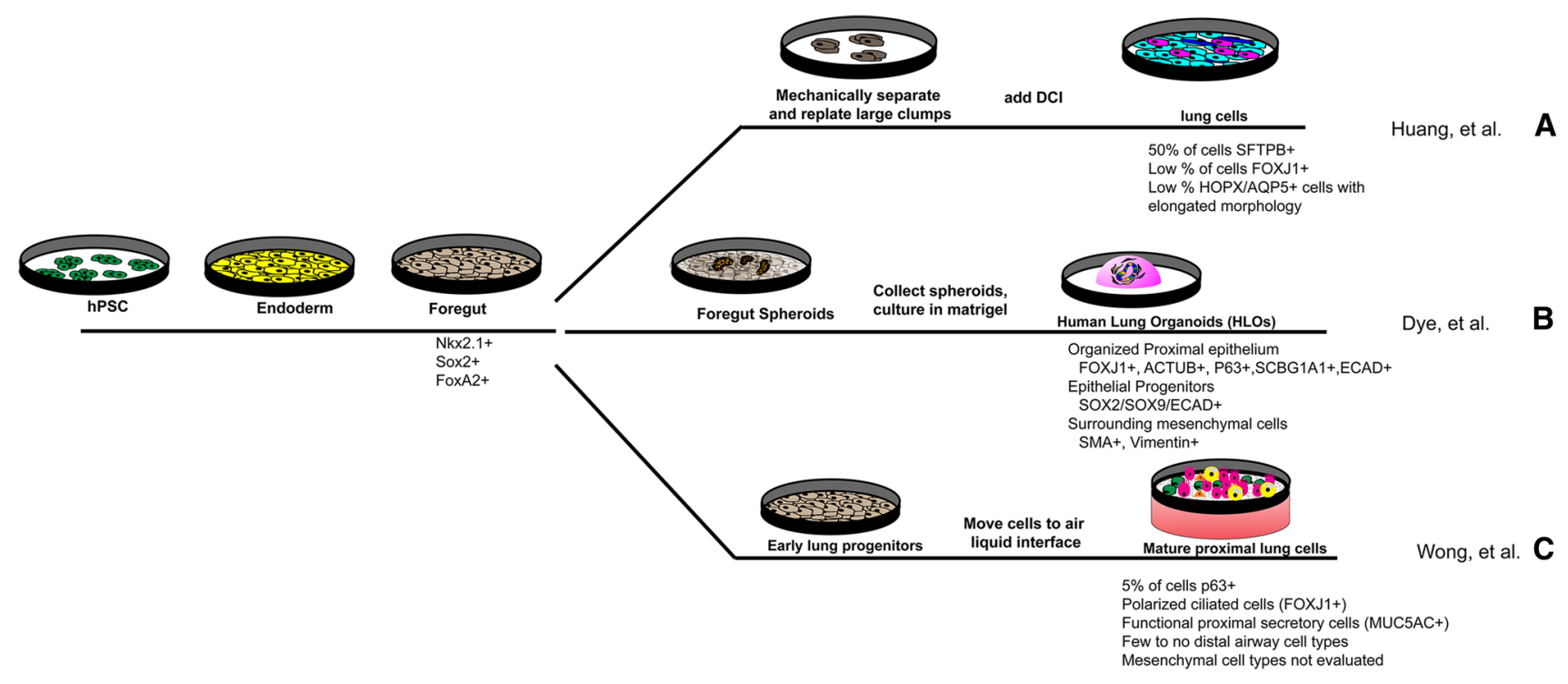

Fig. 2 The majority of protocols to derive lung cell types from hPSCs have taken a directed differentiation approach. hPSCs are first treated with growth factors, including ActivinA, to derive endoderm, which is further treated to become anterior foregut endoderm. Anterior foregut endoderm cells are marked by Nkx2.1, FoxA2, and Sox 2 transcription factors. Different groups have used various methods to derive lung cell types after this stage. a Huang et al. grew foregut cultures for 15 days, after which cells were broken up and remaining large clumps were re-plated. On day 25 , cells were treated with a DCI cocktail, which has been shown to induce alveolar specific cell-type gene expression in vitro [34, 94]. After 48 days in monolayer culture, the majority of cells expressed the AECII marker, SFTPB. A low number of cells also expressed the proximal ciliated cell marker FOXJ1, and some cells expressed markers for mature AECI (HOPX, AQP5) cells and exhibited elongated nuclei. b Dye et al. treated foregut cultures with FGF4 and Wnt to induce threedimensional foregut spheroids, which were cultured in a matrigel

regions of the lung organoid, but true alveolar sac-like structures were not observed [39•].

Thus, one of the hurdles that remain in hPSC-derived lung differentiation is to successfully recapitulate alveolar structure in vitro. Alveolar-like structures have been derived from primary human AECII cells co-cultured with fetal human lung fibroblast cell lines forming alveolar spheres (alveolospheres). The cultures consist of phenotypic AECI and AECII cells demonstrating proof of concept that achieving such three-dimensional alveolar structure is possible [99]. Similar approaches have been attempted with hPSC-derived tissues, where the cell surface marker carboxypeptidase M (CPM) was used to enrich NKX2.1+ progenitors using fluorescent-activated cell sorting (FACS). CPM-purified lung progenitors were cultured in a three-dimensional extracellular matrix along with FGF7, DCI, and a fetal human lung fibroblast cell line. This resulted in three-dimensional spheres composed of cells expressing AECI and AECII markers, yet proper AECI morphology was not demonstrated [41•]. Although droplet. These lung organoids persisted in culture for over 100 days and contained an organized epithelium containing cells positive for proximal airway markers FOXJ1/ACTUB (ciliated cells), p63 (basal stem cells), SCBGA1A1 (club cells), and surrounding mesenchymal tissue positive for smooth muscle (SMA) and vimentin (VIM). Additionally, organoids contained some cells that stained positive for AECI and AECII cell makers, but organized alveolar structures were not observed. c Wong et al. used multiple growth factor cocktails over the course of 35 days to induce lung progenitor cells in a monolayer culture. After roughly 5 weeks in culture, cells were moved to an airliquid interface environment, which resulted in maturation of lung cells. The majority of cells expressed mature proximal cell markers for ciliated cells (FOXJ1) or goblet cells (MUC5AC), and cells exhibited distinct polarization, with the basal side of cells facing the media and the apical side of cells facing the air, similar to the in vivo polarization of lung cells with apical surfaces facing the airway lumen

effective, one of the drawbacks to this approach is that it is unclear if the human fetal feeder cells are providing a physical niche through cell-cell contact, and/or if they secrete important factors $[41 \bullet, 99]$. While several groups have shown success obtaining distal epithelial cell types in vitro, additional work is needed to better understand the mechanisms that control AECI and AECII differentiation to improve upon the efficiency of the differentiation and deriving alveolar structure.

\section{Proximal Airway Differentiation}

In the developing embryo, a variety of factors are important for promoting proximal airway fate in mouse lungs [100]. As development progresses, the Sox 9/Id2/Nmyc bud tips differentiate into proximal tissue and express the marker Sox2. The multipotent Sox2+ airway population will give rise to neuroendocrine, club, ciliated, goblet, and basal cells [28]. Activation of Notch signaling promotes 
Sox $2+$ cells to differentiate into secretory cells, whereas inhibition of Notch signaling promotes ciliated cells and neuroendocrine cell fates [101-103]. During late lung development and adult homeostasis, the Fgf signaling ligand Fgf18 is necessary for maintaining basal stem cells in the proximal airways, and Notch signaling acts to control the balance of secretory and ciliated cell types $[5,9$, $100,104,105]$

In order to differentiate proximal airway cell types from hPSC-derived endoderm, approaches to both promote proximal airway differentiation and to reduce distal airway differentiation have been taken (Fig. 2c). In order to steer cells away from a distal fate, BMP4 concentrations were reduced and FGF18 was added to promote proximal cell fates, an approach that resulted in over $50 \%$ of the cells expressing the basal stem cell marker P63. To further mature proximal cell types, cultures were moved to an airliquid interface (ALI) resulting in an increase in mature airway cells and polarization of the airway epithelium. The cells on the ALI expressed markers for goblet, club, and ciliated cells along with basal cells [36॰]. Together, these results suggest that foregut endoderm can be directed to a proximal lung fate by a combination of reducing factors that promoted distal fate and adding factors that promoted proximal fate. However, the role of the polarizing ALI environment and the undefined growth factors present in commercially purchased ALI media in this approach leaves room for further exploration.

In addition to the ALI platform, three-dimensional airway cultures have been generated from hPSCs, applying similar concepts used to derive "bronchosphere" from human and mouse primary basal cells, an approach first establishing that human basal cells can self-renew and generate ciliated and club cells in vitro [5]. Successful growth of bronchospheres from hPSCs has been achieved using purified $\mathrm{CPM}+$ foregut progenitor cells, similar to the approach used for alveolarspheres [40•, 41•]. Using commercially available ALI media and Notch inhibition, the sorted CPM+ population formed spheres in a threedimensional matrix from single cells. Spheres consisted of mostly ciliated cells interspersed with neuroendocrine cells with even fewer basal cells and secretory cells. These hPSC-derived bronchospheres represent the first report of beating ciliated cells that are not derived from a primary cell line; however the cells do not organize into a pseudostratified epithelium, as is the case with the lung in vivo [40॰]. Foregut spheroids grown in a three-dimensional ECM, Matrigel, overlaid with media containing high concentrations of FGF10 also gave rise to proximal airwaylike structures that formed a polarized epithelium organized in a cyst containing a lumen, and surrounded by mesenchyme (Fig. 2b). The epithelium consisted of basal cells close to the mesenchymal tissue, with the adjacent epithelial cell types facing toward the lumen and expressing an early marker of ciliated cells, FOXJ1 [39]. High FGF10 was required for airway-like epithelium to differentiate in lung organoids, and may act to maintain the basal cell population in lung organoids, however, this has not yet been formally tested. Consistent with high FGF10 promoting proximal fates, recent evidence in mice suggests that high levels of ectopic Fgf10 expressed during embryonic development can increase the number of P63+ basal stem cells present in the lung [90]. Interestingly, as has been demonstrated in both lung organoids and other hPSC-derived tissues, including hepatocyte-like, $\beta$ cell-like, intestinal, stomach, and cerebral organoids, even in long-term cultures, the organoids retain a transcriptional profile similar to fetal tissue than to adult tissue $[33,39 \bullet$, 51, 106-108]. Thus, an important unresolved problem in the field is how to overcome this developmental barrier in vitro in order to obtain more mature adult-like tissue. Achieving this goal could require modulation of the physical or chemical environment to induce further maturation of the tissue.

\section{Future Directions: Next Steps for In Vitro Lung Models}

\section{Branching Morphogenesis}

Although the field has achieved success in deriving both proximal and distal cell types from hPSCs in two-dimen-

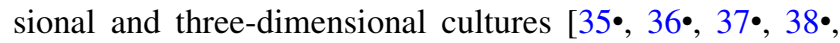
$39 \bullet, 40^{\bullet}, 41^{\bullet}, 42^{\bullet}, 43 \bullet, 44^{\bullet}$, to date, there have been no reports of in vitro human systems that mimic the complex three-dimensional morphology of epithelial branching structures seen in the embryo and bona fide alveolar structures have not been described. Therefore, the development of an hPSC-derived in vitro model of lung branching will be hugely beneficial for furthering our understanding of human lung development and associated pathologies. The development of such a model would provide a critical tool for investigating the complex interactions between the ECM, force gradients, diffusible morphogens, and specific genetic programs active during the development, disease, and repair of the human distal lung regions.

Much of the work in the branching morphogenesis field has focused on cell signaling and genetic factors that regulate this process. However, the physical environment also plays a role in directing branching. It is well established that cues from the ECM, as well as inputs from vascular endothelial cells and neurons play important roles in directing this process. As distal lung buds begin to bifurcate, a thick deposit of ECM is present at the bud cleft, 
while Matrix Metalloprotinases (MMPs) appear to play a role in degrading the ECM around growing bud tips [109]. Recent work has shown that mouse lung buds isolated from the mesenchyme can be cultured in three-dimensional ECM environments of increasing stiffness and as stiffness increases, the buds form more branches, suggesting the mechanical force of the ECM can play a role in directing branching [110]. In addition to mechanical influences, vascular endothelial cells play a role in early branching morphogenesis and in maintaining lung epithelial stem cells in vivo [99, 111-114]. Similarly, migrating neural crest cells will invade the early lung and become neurons, which are necessary for proper branching morphogenesis [115-117]. Together, this evidence from animal models suggests that mechanical forces, as well as inputs from vascular endothelial cells and neuronal innervation all play essential roles in defining and maintaining the lung architecture during lung branching. Yet, so far, these critical physical regulators of lung organogenesis have eluded translation into hPSC-derived in vitro models. Careful attention to defining an optimal physical environment and cues from closely associated non-lung cell types may improve the complex architecture and patterning of in vitro hPSC-derived lung tissue cultures in the future, and will be a critical next step to advancing models of human lung development, disease, and regeneration.

In addition to improving our understanding of the physical environment of the developing lung, detailed investigation into the types, concentrations, and gradients of diffusible morphogen signals that promote a distal versus proximal lung fate, and tissue engineering approaches to appropriately deliver regulated morphogen gradients, may also improve existing techniques for generating hPSCderived models of the human lung.

\section{Functional Testing}

Although many cell markers exist to identify specific lung cell types derived from hPSCs, the functional studies implemented thus far have been limited to surfactant protein production and uptake for AECII cells [38] and recording ciliated cell movement [40•]. These tests show a very specific function for limited cell types in the lungs. Unfortunately, there is no comprehensive functional test for in vitro lung cultures. In rats, decellularized lung matrices have been seeded with neonatal rat lung epithelial plus vasculature and were functionally assessed for their ability to sustain life for a short period of time [118, 119]. In addition, human decellularized lung matrices have been hooked up to a bioreactor to create an environment for fluid and air exchange to take place [118, 120-122]. However, it is unclear if reseeding an entire adult human lung matrix is feasible for such bioreactor studies, and either more efficient methods for reseeding need to be developed, or more realistic lung structures with branched tubular networks need to be differentiated de novo.

Along with testing overall organ function, cell-specific tests will also be necessary to test lung cellular function. Cellular tests for AECII and ciliated cells have been demonstrated [38•, 40•], but there is still no functional test for AECI or secretory cells. AECI cells function mainly to exchange gas; therefore, in vitro methods to measure gas diffusion in a highly sensitive fashion may need to be developed. For secretory cells, sensitive methods to stimulate and measure proteins released by secretory cells (goblet, club cells) will also be highly valuable for establishing function. Measuring protein secretion of single cells in vitro has been established, but ideally the goal would be to measure protein secretion within three-dimensional airway structures [123, 124]. Taken together, two types of functional tests need to be implemented: the first to test the overall lung function of gas exchange and airway movement and the second to hone in on a specific cellular function.

\section{Conclusions}

Currently, the majority of hPSC-derived human lung models are generated by treating hPSCs with a series of growth factor combinations that attempt to mimic the signaling milieu observed in vivo to direct differentiation to lung-specific cell types. These methods have resulted in a diverse range of model systems and this approach has been effective in generating many lung cell types and relevant lung architecture. Yet, gold standard functional tests need to be established to validate the diverse findings using different methodologies. These tests will ideally include both cellular function and assessment of tissue/ organ-level function. In just the past few years, various human lung models have been derived that can be applied to study lung development, disease, and tissue regeneration. However, mimicking the in vivo environment of the lung by including other tissues including mesenchyme, vasculature, and neurons will make for an even more realistic human model moving forward.

Acknowledgments JRS is supported by the NIH-NHLBI (R01 HL119215). AJM is supported by the NIH Cellular and Molecular Biology training grant at Michigan (T32 GM007315). BRD is supported by a Graduate Fellowship from the University of Michigan Rackham graduate school.

\section{Compliance with Ethics Guidelines}

Conflict of Interest Alyssa J. Miller has no conflict of interest to declare. Jason R. Spence and Briana R. Dye have a patent pending related to the methods to generate human lung organoids (U.S. Provisional Patent Application No. 62/151,238). 
Human and Animal Rights and Informed Consent All published work conducted by the authors that has been cited in this review also conforms to ethical guidelines for use of animals, human subjects and human pluripotent stem cells, and was conducted with the appropriate institutional approval and oversight.

Open Access This article is distributed under the terms of the Creative Commons Attribution 4.0 International License (http://creativecommons. org/licenses/by/4.0/), which permits unrestricted use, distribution, and reproduction in any medium, provided you give appropriate credit to the original author(s) and the source, provide a link to the Creative Commons license, and indicate if changes were made.

\section{References}

Papers of particular interest, published recently, have been highlighted as:

- Of importance

-• Of major importance

1. Mercer RR, Russell ML, Roggli VL, Crapo JD (1994) Cell number and distribution in human and rat airways. Am J Respir Cell Mol Biol 10:613-624. doi:10.1165/ajrcmb.10.6.8003339

2. Wolff RK (1986) Effects of airborne pollutants on mucociliary clearance. Environ Health Perspect 66:223-237

3. Schum M, Yeh HC (1980) Theoretical evaluation of aerosol deposition in anatomical models of mammalian lung airways. Bull Math Biol 42:1-15

4. Rogers DF (1994) Airway goblet cells: responsive and adaptable front-line defenders. Eur Respir J 7:1690-1706

5. Rock JR, Onaitis MW, Rawlins EL et al (2009) Basal cells as stem cells of the mouse trachea and human airway epithelium. Proc Natl Acad Sci 106:12771-12775. doi:10.1073/pnas. 0906850106

6. Hackett T-L, Shaheen F, Johnson A et al (2008) Characterization of side population cells from human airway epithelium. Stem Cells 26:2576-2585. doi:10.1634/stemcells.2008-0171

7. Hong KU, Reynolds SD, Watkins S et al (2004) Basal cells are a multipotent progenitor capable of renewing the bronchial epithelium. AJPA 164:577-588. doi:10.1016/S0002-9440(10)63147-1

8. Boers JE, Ambergen AW, Thunnissen FB (1998) Number and proliferation of basal and parabasal cells in normal human airway epithelium. Am J Respir Crit Care Med 157:2000-2006. doi:10.1164/ajrccm.157.6.9707011

9. Pardo-Saganta A, Law BM, Tata PR et al (2015) Injury induces direct lineage segregation of functionally distinct airway basal stem/progenitor cell subpopulations. Cell Stem Cell 16:184197. doi:10.1016/j.stem.2015.01.002

10. Nakajima M, Kawanami O, Jin E et al (1998) Immunohistochemical and ultrastructural studies of basal cells, Clara cells and bronchiolar cuboidal cells in normal human airways. Pathol Int 48:944-953

11. Evans MJ, Van Winkle LS, Fanucchi MV, Plopper CG (2001) Cellular and molecular characteristics of basal cells in airway epithelium. Exp Lung Res 27:401-415

12. Boers JE, Ambergen AW, Thunnissen FB (1999) Number and proliferation of clara cells in normal human airway epithelium. Am J Respir Crit Care Med 159:1585-1591. doi:10.1164/ ajrccm.159.5.9806044

13. Linnoila RI (2006) Functional facets of the pulmonary neuroendocrine system. Lab Invest 86:425-444. doi:10.1038/ labinvest. 3700412
14. Domnik NJ, Cutz E (2011) Pulmonary neuroepithelial bodies as airway sensors: putative role in the generation of dyspnea. Curr Opin Pharmacol 11:211-217. doi:10.1016/j.coph.2011.04.003

15. Song H, Yao E, Lin C et al (2012) Functional characterization of pulmonary neuroendocrine cells in lung development, injury, and tumorigenesis. Proc Natl Acad Sci 109:17531-17536. doi:10.1073/pnas.1207238109

16. Branchfield K, Nantie L, Verheyden JM et al (2016) Pulmonary neuroendocrine cells function as airway sensors to control lung immune response. Science. doi:10.1126/science.aad7969

17. Williams MC (2003) Alveolar type I cells: molecular phenotype and development. Annu Rev Physiol 65:669-695. doi:10.1146/ annurev.physiol.65.092101.142446

18. Féréol S, Fodil R, Pelle G et al (2008) Cell mechanics of alveolar epithelial cells (AECs) and macrophages (AMs). Respir Physiol Neurobiol 163:3-16. doi:10.1016/j.resp.2008.04.018

19. Yang J, Hernandez BJ, Martinez Alanis D et al (2016) The development and plasticity of alveolar type 1 cells. Development 143:54-65. doi:10.1242/dev.130005

20. Branchfield K, Li R, Lungova V et al (2016) A three-dimensional study of alveologenesis in mouse lung. Dev Biol 409:429-441. doi:10.1016/j.ydbio.2015.11.017

21. Castranova V, Rabovsky J, Tucker JH, Miles PR (1988) The alveolar type II epithelial cell: a multifunctional pneumocyte. Toxicol Appl Pharmacol 93:472-483

22. Fehrenbach H (2001) Alveolar epithelial type II cell: defender of the alveolus revisited. Respir Res 2:33-46

23. Wells JM, Spence JR (2014) How to make an intestine. Development 141:752-760. doi:10.1242/dev.097386

24. Spence JR, Wells JM (2007) Translational embryology: using embryonic principles to generate pancreatic endocrine cells from embryonic stem cells. Dev Dyn 236:3218-3227. doi:10. 1002/dvdy.21366

25. Finkbeiner SR, Spence JR (2013) A gutsy task: generating intestinal tissue from human pluripotent stem cells. Dig Dis Sci 58:1176-1184. doi:10.1007/s10620-013-2620-2

26. Zorn AM, Wells JM (2009) Vertebrate endoderm development and organ formation. Annu Rev Cell Dev Biol 25:221-251. doi:10.1146/annurev.cellbio.042308.113344

27. Metzger RJ, Klein OD, Martin GR, Krasnow MA (2008) The branching programme of mouse lung development. Nature 453:745-750. doi:10.1038/nature07005

28. Morrisey EE, Hogan BLM (2010) Preparing for the first breath: genetic and cellular mechanisms in lung development. Dev Cell 18:8-23. doi:10.1016/j.devcel.2009.12.010

29. Rawlins EL (2010) The building blocks of mammalian lung development. Dev Dyn 240:463-476. doi:10.1002/dvdy.22482

30. • D'Amour KA, Agulnick AD, Eliazer S et al (2005) Efficient differentiation of human embryonic stem cells to definitive endoderm. Nat Biotechnol 23:1534-1541. doi:10.1038/nbt1163. This was the first report to show efficient endoderm differentiation from human embryonic stem cells

31. Cai J, Zhao Y, Liu Y et al (2007) Directed differentiation of human embryonic stem cells into functional hepatic cells. Hepatology 45:1229-1239. doi:10.1002/hep.21582

32. Kroon E, Martinson LA, Kadoya K et al (2008) Pancreatic endoderm derived from human embryonic stem cells generates glucose-responsive insulin-secreting cells in vivo. Nat Biotechnol 26:443-452. doi:10.1038/nbt1393

33. Si-Tayeb K, Noto FK, Nagaoka M et al (2010) Highly efficient generation of human hepatocyte-like cells from induced pluripotent stem cells. Hepatology 51:297-305. doi:10.1002/ hep. 23354

34. Spence JR, Mayhew CN, Rankin SA et al (2011) Directed differentiation of human pluripotent stem cells into intestinal tissue in vitro. Nature 470:105-109. doi:10.1038/nature09691 
35. • Longmire TA, Ikonomou L, Hawkins F et al (2012) Efficient derivation of purified lung and thyroid progenitors from embryonic stem cells. Cell Stem Cell 10:398-411. doi:10.1016/ j.stem.2012.01.019. This study demonstrated that mouse ESCs could be directed into lung lineages. $m E S C$-derived endoderm was patterned into anterior foregut using BMP/TGFB inhibition, and then further specified into lung or thyroid lineages

36. - Wong AP, Bear CE, Chin S et al (2012) Directed differentiation of human pluripotent stem cells into mature airway epithelia expressing functional CFTR protein. Nat Biotechnol 30:875-881. doi:10.1038/nbt.2328. The authors used air-liquid interface cell culture to further differentiate the lung progenitors to mature airway cells that were polarized

37. • Mou H, Zhao R, Sherwood R et al (2012) Generation of multipotent lung and airway progenitors from mouse ESCs and patientspecific cystic fibrosis iPSCs. Cell Stem Cell 10:385-397. doi:10. 1016/j.stem.2012.01.018. This study defined methods to generate both mouse and human lung progenitors from pluripotent stem cells, and was one of the first studies to report the use of patient specific iPSC lines to study a lung disease

38. • Huang SXL, Islam MN, O'Neill J, et al (2013) Efficient generation of lung and airway epithelial cells from human pluripotent stem cells. Nat Biotechnol doi: 10.1038/nbt.2754. By re-plating the cultures and changing growth factor combinations caused the cultures to express mature cell types including surfactant producing AECIIs

39. - Dye BR, Hill DR, Ferguson MA et al (2015) In vitro generation of human pluripotent stem cell derived lung organoids. Elife. doi:10.7554/eLife.05098. This was the first report to generate a three-dimensional model that possessed airway-like structures called human lung organoids. The lung organoids expressed pockets of distal epithelial cells and proximal airwaylike structures surrounded by mesenchyme

40. • Konishi S, Gotoh S, Tateishi K et al (2016) Directed induction of functional multi-ciliated cells in proximal airway epithelial spheroids from human pluripotent stem cells. Stem Cell Rep 6:18-25. doi:10.1016/j.stemcr.2015.11.010. This study identified a cell surface marker allowing the purification of lung progenitor cells. Purified lung progenitor cells generated bronchospheres, and demonstrated mucociliary function

41. - Gotoh S, Ito I, Nagasaki T et al (2014) Generation of alveolar epithelial spheroids via isolated progenitor cells from human pluripotent stem cells. Stem Cell Rep 3:394-403. doi:10.1016/j. stemcr.2014.07.005. This study identified a cell surface marker allowing the purification of hPSC-derived lung progenitor cells. Purified lung progenitor cells were further differentiated into alveolus-like structures

42. - Ghaedi M, Mendez JJ, Bove PF et al (2014) Alveolar epithelial differentiation of human induced pluripotent stem cells in a rotating bioreactor. Biomaterials 35:699-710. doi:10.1016/j. biomaterials.2013.10.018. Tissue-derived and iPSC-derived alveolar type 2 cells were cultured in rotating bioreactor that mimicked in vivo respiratory conditions

43. - Ghaedi M, Calle EA, Mendez JJ et al (2013) Human iPS cellderived alveolar epithelium repopulates lung extracellular matrix. J Clin Invest 123:4950-4962. doi:10.1172/JCI68793. hPSC were directed to differentiate into alveolar type II cells with high purity. ATII cells were seeded on acellular rat or human lung matrix, and were shown to proliferate and differentiate into alveolar cell types on the matrices

44. - Hannan NRF, Sampaziotis F, Segeritz C-P et al (2015) Generation of distal airway epithelium from multipotent human foregut stem cells. Stem Cells Dev 24:1680-1690. doi:10.1089/ scd.2014.0512. This study demonstrated how to generate distal airway-like epithelium from hPSCs, and also showed some data with hPSC-derived lung organoids
45. - Firth AL, Dargitz CT, Qualls SJ et al (2014) Generation of multiciliated cells in functional airway epithelia from human induced pluripotent stem cells. Proc Natl Acad Sci 111:E1723E1730. doi:10.1073/pnas.1403470111. This report demonstrated methods to generate endoderm, foregut endoderm, followed by differentiation of monolayers of upper airway-like pseudostratified epithelium that possessed abundant multiciliated cells

46. Schier AF (2003) Nodal signaling in vertebrate development. Annu Rev Cell Dev Biol 19:589-621. doi:10.1146/annurev. cellbio.19.041603.094522

47. Schier AF, Shen MM (2000) Nodal signalling in vertebrate development. Nature 403:385-389. doi:10.1038/35000126

48. Aoki TO, David NB, Minchiotti G et al (2002) Molecular integration of casanova in the Nodal signalling pathway controlling endoderm formation. Development 129:275-286

49. Shen MM (2007) Nodal signaling: developmental roles and regulation. Development 134:1023-1034. doi:10.1242/dev.000166

50. Hagos EG, Dougan ST (2007) Time-dependent patterning of the mesoderm and endoderm by Nodal signals in zebrafish. BMC Dev Biol 7:22. doi:10.1186/1471-213X-7-22

51. McCracken KW, Catá EM, Crawford CM et al (2014) Modelling human development and disease in pluripotent stem-cellderived gastric organoids. Nature 516:400-404. doi:10.1038/ nature 13863

52. Rossant J, Tam PPL (2009) Blastocyst lineage formation, early embryonic asymmetries and axis patterning in the mouse. Development 136:701-713. doi:10.1242/dev.017178

53. Yamamoto M, Saijoh Y, Perea-Gomez A et al (2004) Nodal antagonists regulate formation of the anteroposterior axis of the mouse embryo. Nature 428:387-392. doi:10.1038/nature02418

54. Perea-Gomez A, Vella FDJ, Shawlot W et al (2002) Nodal antagonists in the anterior visceral endoderm prevent the formation of multiple primitive streaks. Dev Cell 3:745-756

55. Tiso N, Filippi A, Pauls S et al (2002) BMP signalling regulates anteroposterior endoderm patterning in zebrafish. Mech Dev 118:29-37

56. Li Y, Rankin SA, Sinner D et al (2008) Sfrp5 coordinates foregut specification and morphogenesis by antagonizing both canonical and noncanonical Wnt11 signaling. Genes Dev 22:3050-3063. doi:10.1101/gad.1687308

57. • Green MD, Chen A, Nostro M-C et al (2011) Generation of anterior foregut endoderm from human embryonic and induced pluripotent stem cells. Nat Biotechnol 29:267-272. doi:10.1038/ nbt.1788. This study identified efficient methods to pattern hPSC-derived endoderm into foregut endoderm. This general approach has been adopted by many in order to derive anterior foregut lineages including lung tissue

58. McCracken KW, Howell JC, Wells JM, Spence JR (2011) Generating human intestinal tissue from pluripotent stem cells in vitro. Nat Protoc 6:1920-1928. doi:10.1038/nprot.2011.410

59. Schlessinger K, Hall A, Tolwinski N (2009) Wnt signaling pathways meet Rho GTPases. Genes Dev 23:265-277. doi:10. 1101/gad.1760809

60. Li S, Muneoka K (1999) Cell migration and chick limb development: chemotactic action of FGF-4 and the AER. Developmental Biology 211:335-347. doi:10.1006/dbio.1999.9317

61. Sun X, Meyers EN, Lewandoski M, Martin GR (1999) Targeted disruption of Fgf8 causes failure of cell migration in the gastrulating mouse embryo. Genes Dev 13:1834-1846

62. Crump JG, Maves L, Lawson ND et al (2004) An essential role for Fgfs in endodermal pouch formation influences later craniofacial skeletal patterning. Development 131:5703-5716. doi:10.1242/dev.01444

63. Sherwood RI, Maehr R, Mazzoni EO, Melton DA (2011) Wnt signaling specifies and patterns intestinal endoderm. Mech Dev. doi:10.1016/j.mod.2011.07.005 
64. Lazzaro D, Price M, de Felice M, Di Lauro R (1991) The transcription factor TTF-1 is expressed at the onset of thyroid and lung morphogenesis and in restricted regions of the foetal brain. Development 113:1093-1104

65. Minoo P, Su G, Drum H et al (1999) Defects in tracheoesophageal and lung morphogenesis in $\mathrm{Nkx} 2.1(-/-)$ mouse embryos. Dev Biol 209:60-71. doi:10.1006/dbio.1999.9234

66. Rankin SA, Zorn AM (2014) Gene regulatory networks governing lung specification. J Cell Biochem 115:1343-1350. doi: $10.1002 /$ jcb. 24810

67. Domyan ET, Ferretti E, Throckmorton K et al (2011) Signaling through BMP receptors promotes respiratory identity in the foregut via repression of Sox2. Development 138:971-981. doi:10.1242/dev.053694

68. Serls AE, Doherty S, Parvatiyar P et al (2005) Different thresholds of fibroblast growth factors pattern the ventral foregut into liver and lung. Development 132:35-47. doi:10.1242/dev. 01570

69. Min H, Danilenko DM, Scully SA et al (1998) Fgf-10 is required for both limb and lung development and exhibits striking functional similarity to Drosophila branchless. Genes Dev 12:3156-3161

70. Sekine K, Ohuchi H, Fujiwara M et al (1999) Fgf10 is essential for limb and lung formation. Nat Genet 21:138-141. doi:10. $1038 / 5096$

71. Shifley ET, Kenny AP, Rankin SA, Zorn AM (2012) Prolonged FGF signaling is necessary for lung and liver induction in Xenopus. BMC Dev Biol 12:27. doi:10.1186/1471-213X-12-27

72. Goss AM, Tian Y, Tsukiyama T et al (2009) Wnt2/2b and $\beta$ catenin signaling are necessary and sufficient to specify lung progenitors in the foregut. Dev Cell 17:290-298. doi:10.1016/j. devcel.2009.06.005

73. Harris-Johnson KS, Domyan ET, Vezina CM, Sun X $\beta$-Catenin promotes respiratory progenitor identity in mouse foregut

74. Rankin SA, Gallas AL, Neto A et al (2012) Suppression of Bmp4 signaling by the zinc-finger repressors Osr1 and Osr2 is required for Wnt/-catenin-mediated lung specification in Xenopus. Development 139:3010-3020. doi:10.1242/dev. 078220

75. Miller MF, Cohen ED, Baggs JE et al (2012) Wnt ligands signal in a cooperative manner to promote foregut organogenesis. Proc Natl Acad Sci 109:15348-15353. doi:10.1073/pnas.1201583109

76. Chen F, Cao Y, Qian J et al (2010) A retinoic acid-dependent network in the foregut controls formation of the mouse lung primordium. J Clin Invest 120:2040-2048. doi:10.1172/ JCI40253

77. Chen F, Desai TJ, Qian J et al (2007) Inhibition of Tgf beta signaling by endogenous retinoic acid is essential for primary lung bud induction. Development 134:2969-2979. doi:10.1242/ dev.006221

78. Motoyama J, Liu J, Mo R et al (1998) Essential function of Gli2 and Gli3 in the formation of lung, trachea and oesophagus. Nat Genet 20:54-57. doi:10.1038/1711

79. Rockich BE, Hrycaj SM, Shih HP et al (2013) Sox9 plays multiple roles in the lung epithelium during branching morphogenesis. Proc Natl Acad Sci 110:E4456-E4464. doi:10.1073/ pnas. 1311847110

80. Okubo T (2005) Nmyc plays an essential role during lung development as a dosage-sensitive regulator of progenitor cell proliferation and differentiation. Development 132:1363-1374. doi:10.1242/dev.01678

81. Perl AKT, Kist R, Shan Z et al (2005) Normal lung development and function after Sox 9 inactivation in the respiratory epithelium. Genesis 41:23-32. doi:10.1002/gene.20093

82. Rawlins EL, Clark CP, Xue Y, Hogan BLM (2009) The Id2+ distal tip lung epithelium contains individual multipotent embryonic progenitor cells. Development 136:3741-3745. doi: $10.1242 /$ dev. 037317

83. Weaver M, Yingling JM, Dunn NR et al (1999) Bmp signaling regulates proximal-distal differentiation of endoderm in mouse lung development. Development 126:4005-4015

84. Mucenski ML, Wert SE, Nation JM et al (2003) beta-Catenin is required for specification of proximal/distal cell fate during lung morphogenesis. J Biol Chem 278:40231-40238. doi:10.1074/ jbc.M305892200

85. De Langhe SP, Reynolds SD (2008) Wnt signaling in lung organogenesis. Organogenesis 4:100-108

86. Kadzik RS, Cohen ED, Morley MP et al (2014) Wnt ligand/ Frizzled 2 receptor signaling regulates tube shape and branchpoint formation in the lung through control of epithelial cell shape. Proc Natl Acad Sci 111:12444-12449. doi:10.1073/pnas. 1406639111

87. Rajagopal J, Carroll TJ, Guseh JS et al (2008) Wnt7b stimulates embryonic lung growth by coordinately increasing the replication of epithelium and mesenchyme. Development 135:16251634. doi:10.1242/dev.015495

88. Shu W, Guttentag S, Wang Z et al (2005) Wnt/ $\beta$-catenin signaling acts upstream of N-myc, BMP4, and FGF signaling to regulate proximal-distal patterning in the lung. Dev Biol 283: 226-239. doi:10.1016/j.ydbio.2005.04.014

89. Bellusci S, Grindley J, Emoto H et al (1997) Fibroblast growth factor 10 (FGF10) and branching morphogenesis in the embryonic mouse lung. Development 124:4867-4878

90. Volckaert T, Campbell A, Dill E et al (2013) Localized Fgf10 expression is not required for lung branching morphogenesis but prevents differentiation of epithelial progenitors. Development 140:3731-3742. doi:10.1242/dev.096560

91. Shiratori M, Oshika E, Ung LP et al (1996) Keratinocyte growth factor and embryonic rat lung morphogenesis. Am J Respir Cell Mol Biol 15:328-338. doi:10.1165/ajrcmb.15.3.8810636

92. Yano T, Mason RJ, Pan T et al (2000) KGF regulates pulmonary epithelial proliferation and surfactant protein gene expression in adult rat lung. Am J Physiol Lung Cell Mol Physiol 279:L1146L1158

93. Nyeng P, Norgaard GA, Kobberup S, Jensen J (2008) FGF10 maintains distal lung bud epithelium and excessive signaling leads to progenitor state arrest, distalization, and goblet cell metaplasia. BMC Dev Biol 8:2. doi:10.1186/1471-213X-8-2

94. Desai TJ, Chen F, Lü J et al (2006) Distinct roles for retinoic acid receptors alpha and beta in early lung morphogenesis. Dev Biol 291:12-24. doi:10.1016/j.ydbio.2005.10.045

95. Desai TJ, Brownfield DG, Krasnow MA (2014) Alveolar progenitor and stem cells in lung development, renewal and cancer. Nature. doi:10.1038/nature12930

96. Gonzales LW, Guttentag SH, Wade KC et al (2002) Differentiation of human pulmonary type II cells in vitro by glucocorticoid plus cAMP. Am J Physiol Lung Cell Mol Physiol 283:L940-L951. doi:10.1152/ajplung.00127.2002

97. Crowley P, Chalmers I, Keirse MJ (1990) The effects of corticosteroid administration before preterm delivery: an overview of the evidence from controlled trials. $\mathrm{Br} \mathrm{J}$ Obstet Gynaecol 97:11-25

98. Wapner R (2004) Antenatal corticosteroids: we continue to learn. Am J Obstet Gynecol 190:875. doi:10.1016/j.ajog.2004. 01.045

99. Barkauskas CE, Cronce MJ, Rackley CR et al (2013) Type 2 alveolar cells are stem cells in adult lung. J Clin Invest 123:3025-3036. doi:10.1172/JCI68782

100. Whitsett JA, Clark JC, Picard L et al (2002) Fibroblast growth factor 18 influences proximal programming during lung morphogenesis. J Biol Chem 277:22743-22749. doi:10.1074/jbc. M202253200 
101. Guseh JS, Bores SA, Stanger BZ et al (2009) Notch signaling promotes airway mucous metaplasia and inhibits alveolar development. Development 136:1751-1759. doi:10.1242/dev.029249

102. Morimoto M, Liu Z, Cheng H-T et al (2010) Canonical Notch signaling in the developing lung is required for determination of arterial smooth muscle cells and selection of Clara versus ciliated cell fate. J Cell Sci 123:213-224. doi:10.1242/jcs.058669

103. Zhang S, Loch AJ, Radtke F et al (2013) Jagged1 is the major regulator of Notch-dependent cell fate in proximal airways. Dev Dyn 242:678-686. doi:10.1002/dvdy.23965

104. Tadokoro T, Wang Y, Barak LS et al (2014) IL-6/STAT3 promotes regeneration of airway ciliated cells from basal stem cells. Proc Natl Acad Sci 111:E3641-E3649. doi:10.1073/pnas. 1409781111

105. Rock JR, Gao X, Xue Y et al (2011) Notch-dependent differentiation of adult airway basal stem cells. Cell Stem Cell 8:639-648. doi:10.1016/j.stem.2011.04.003

106. Hrvatin S, O'Donnell CW, Deng F et al (2014) Differentiated human stem cells resemble fetal, not adult, $\beta$ cells. Proc Natl Acad Sci 111:3038-3043. doi:10.1073/pnas.1400709111

107. Finkbeiner SR, Hill DR, Altheim CH et al (2015) Transcriptome-wide analysis reveals hallmarks of human intestine development and maturation in vitro and in vivo. Stem Cell Rep. doi:10.1016/j.stemcr.2015.04.010

108. Camp JG, Badsha F, Florio M et al (2015) Human cerebral organoids recapitulate gene expression programs of fetal neocortex development. Proc Natl Acad Sci 112:15672-15677. doi:10.1073/pnas.1520760112

109. Kheradmand F, Rishi K, Werb Z (2002) Signaling through the EGF receptor controls lung morphogenesis in part by regulating MT1-MMP-mediated activation of gelatinase A/MMP2. J Cell Sci 115:839-848

110. Kim HY, Nelson CM (2012) Extracellular matrix and cytoskeletal dynamics during branching morphogenesis. Organogenesis 8:56-64

111. van Tuyl M, Groenman F, Wang J et al (2007) Angiogenic factors stimulate tubular branching morphogenesis of sonic hedgehog-deficient lungs. Dev Biol 303:514-526. doi:10.1016/j. ydbio.2006.11.029

112. Del Moral P-M, Sala FG, Tefft D et al (2006) VEGF-A signaling through Flk-1 is a critical facilitator of early embryonic lung epithelial to endothelial crosstalk and branching morphogenesis. Dev Biol 290:177-188. doi:10.1016/j.ydbio.2005.11.022
113. Franzdóttir SR, Axelsson IT, Arason AJ et al (2010) Airway branching morphogenesis in three dimensional culture. Respir Res 11:162. doi:10.1186/1465-9921-11-162

114. Lee J-H, Bhang DH, Beede A et al (2014) Lung stem cell differentiation in mice directed by endothelial cells via a BMP4NFATc1-thrombospondin-1 axis. Cell 156:440-455. doi:10. 1016/j.cell.2013.12.039

115. Bower DV, Lee H-K, Lansford R et al (2014) Airway branching has conserved needs for local parasympathetic innervation but not neurotransmission. BMC Biol 12:92. doi:10.1186/s12915014-0092-2

116. Freem LJ, Escot S, Tannahill D et al (2010) The intrinsic innervation of the lung is derived from neural crest cells as shown by optical projection tomography in Wnt1-Cre;YFP reporter mice. J Anat 217:651-664. doi:10.1111/j.1469-7580. 2010.01295.x

117. Aven L, Ai X (2013) Mechanisms of respiratory innervation during embryonic development. Organogenesis 9:194-198. doi:10.4161/org.24842

118. Petersen TH, Calle EA, Zhao L et al (2010) Tissue-engineered lungs for in vivo implantation. Science 329:538-541. doi:10. 1126/science. 1189345

119. Panoskaltsis-Mortari A, Weiss DJ (2010) Breathing new life into lung transplantation therapy. Mol Ther 18:1581-1583. doi:10. 1038/mt.2010.177

120. Price AP, England KA, Matson AM et al (2010) Development of a decellularized lung bioreactor system for bioengineering the lung: the matrix reloaded. Tissue Eng Part A 16:2581-2591. doi:10.1089/ten.TEA.2009.0659

121. Song JJ, Kim SS, Liu Z et al (2011) Enhanced in vivo function of bioartificial lungs in rats. Ann Thorac Surg 92:998-1005. doi:10.1016/j.athoracsur.2011.05.018 discussion 1005-6

122. Ott HC, Clippinger B, Conrad C et al (2010) Regeneration and orthotopic transplantation of a bioartificial lung. Nat Med 16:927-933. doi:10.1038/nm.2193

123. Shirasaki Y, Yamagishi M, Suzuki N et al (2014) Real-time single-cell imaging of protein secretion. Sci Rep 4:4736. doi:10. 1038/srep04736

124. Raphael MP, Christodoulides JA, Delehanty JB et al (2013) Quantitative imaging of protein secretions from single cells in real time. Biophys J 105:602-608. doi:10.1016/j.bpj.2013.06. 022 